\title{
Comparison of Platelet-Rich Plasma and Hyaluronic Acid for Treatment of Early Stage Gonarthrosis
}

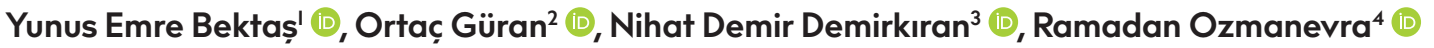 \\ 'Department Orthopaedics and Traumatology, Kilis State Hospital, Kilis, Turkey \\ 2Department of Orthopaedics and Traumatology, Van Özalp State Hospital, Van, Turkey \\ ${ }^{3}$ Department of Orthopaedics and Traumatology, Dumlupınar University, Evliya Çelebi Education and Research Hospital, Kütahya, Turkey \\ ${ }^{4}$ Department of Orthopaedics and Traumatology, University of Kyrenia School of Medicine, Kyrenia, North Cyprus
}

ORCID IDs of the authors: Y.E.B. 0000-000I-555I-6475; O.G. 0000-000I-6485-5807; N.D.D. 0000-0002-0724-9672; R.0̈. 0000-0003-05I54001 . Cite this article as: Bektass YE, Güran O, Demirkan ND, Ozmanevra R. Comparison of Platelet-Rich Plasma and Hyaluronic Acid for
Treatment of Early Stage Gonarthrosis. Cyprus J Med Sci 2020; 5(I): 47-50.

\section{BACKGROUND/AIMS}

This study aimed to compare the outcomes of platelet-rich plasma (PRP) and hyaluronic acid (HA) injections in early-stage gonarthrosis.

\section{MATERIAL and METHODS}

Radiographs were examined retrospectively, and 60 patients with Kellgren Lawrence Stage I, 2, and 3 were included in the study. Twentyeight patients were administered HA, and 32 patients were administered PRP. PRP injections were administered three times in total with two-week intervals, while the HA injection was used once. The initial, l-month, and 6-month follow-up records were obtained. The Western Ontario and McMaster Universities Osteoarthritis Index (WOMAC) (pain, stiffness, physical function) and Visual Analog Scale (VAS) scores were evaluated.

\section{RESULTS}

No statistically significant difference was observed between the HA and PRP groups. When the WOMAC scores were evaluated, it was seen that the function, joint stiffness, and overall WOMAC scores were positively affected in both the groups; however, there was no difference between the two groups.

\section{CONCLUSION}

PRP is a novel treatment option in knee osteoarthritis (OA) management, and an increasing number of clinical studies have shown promising results. Both PRP and HA have positive effect in patients with early gonarthrosis; however, the results indicated no superiority in the PRP group.

Keywords: Gonarthrosis, hyaluronic acid, osteoarthritis, platelet-rich plasma

\section{INTRODUCTION}

Knee osteoarthritis is a very common chronic degenerative disease that can impose significant costs on the health systems (I). This disease is recognized as one of top 10 causes of disability around the world (2). The most common symptoms of knee osteoarthritis are pain and physical limitations that have a significant effect on the individual's quality of life and his/her social and economic activities (3).

Today, drugs, including painkillers, corticosteroids, glucosamine, chondroitin sulfate, and non-steroid anti-inflammatory drugs, are used along with viscosupplementation to relieve pain and symptoms as well as to slow the progression of the arthritis (3). Moreover, intra-articular injections are used as an effective option for the drug therapy of arthritis (4).

Hyaluronic acid (HA) and platelet-rich plasma (PRP) are two treatment methods are currently used in patients with early-stage gonarthrosis and have shown promising results. The present study aimed to compare the outcomes of PRP and HA injections in early-stage gonarthrosis. 


\section{MATERIALS AND METHODS}

Radiographs were examined retrospectively, and 60 patients with Kellgren Lawrence Stage I, 2, and 3 were included in the study. Patients who had undergone PRP and HA therapy from April 2017 to November 2017 at the Dokuz Eylül University Hospital were enrolled in the study. The study protocol was approved by the Ethics Committee at the Dokuz Eylül University. Informed consent was obtained from the patients before the injections were administered, and the WOMAC and VAS scores at follow-ups were used.

The exclusion criteria included previous lower extremity surgery, systemic disorders (diabetes, rheumatic diseases, severe cardiovascular diseases, hematological diseases, and infections), generalized $\mathrm{OA}$, ongoing anticoagulant, or antiaggregant therapy, use of NSAIDs in the 5 days before the injection, hemoglobin values $<1 \mathrm{~g} / \mathrm{dL}$, and platelet values $<150,000 / \mathrm{mm}^{3}$.

Twenty-eight patients were administered HA, and 32 were administered PRP.

PRP injections were administered three times in total with twoweek intervals, while HA was administered as a single, 4-mL injection. For PRP preparation, about $40 \mathrm{~mL}$ of venous blood was centrifuged for 15 min, giving two different layers of red blood cell sediment and plasma. Then, the plasma was separated to the sediment containing platelets. Finally 4-6 mL PRP was obtained.

The injection site on the skin was cleaned with povidone iodine. PRP was injected using a 22-gauge needle while the knee was at $90^{\circ}$ flexion in the sitting position. The inferolateral approach was used and after 15 min of rest, the patients were asked to flex and extend their knees. HA was administered as a single injection with a prepared needle in the same procedure as that used for administering PRP. The initial WOMAC and VAS scores were obtained from the patients' medical records. The initial scores were saved before starting each procedure. No restrictions were placed on the patients, and no complications were observed after the applications.

The initial, I-month, and 6-month follow-up records were obtained. The WOMAC score and VAS scores were evaluated. The WOMAC index consists of 27 questions for three parame-

\section{Main Points:}

- $\quad$ PRP has been accepted as an intra-articular treatment method such as hyaluronic acid in the treatment of knee osteoarthritis especially in the last 10 years.

- $\quad$ There are studies reporting that PRP treatment has satisfactory results, as well as studies concluding that there may be a placebo effect.

- The PRP treatment is considered practically harmless and is being increasingly used. However, higher cost of PRP should be kept on mind and indications should be revised.

- $\quad$ Randomized controlled clinical studies are essential to determine its indications more clearly and to confirm its efficacy and safety. ters, including pain, stiffness, and physical function. Each question is scored from 0 (none) to 4 (extreme). The sum of the scores of the subscales is the total WOMAC score (ranging from 0-108). Higher scores indicated worse conditions. The VAS index was also evaluated. VAS scores range from 0 (no pain) to 10 (worst possible pain).

Data were analyzed by using Statistical Package for the Social Sciences (IBM SPSS Corp.; Armonk, NY, USA) 18.0 Mann Whitney $U$ and Wilcoxon tests were used for the statistical analyses.

\section{RESULTS}

There were 6 men and 22 women in the HA group and 6 men and 26 women in the PRP group. The mean patient age was $63.53 \mathrm{y}$ in the HA group and $63.43 \mathrm{y}$ in the PRP group. The body mass index was $31.48 \mathrm{~kg} / \mathrm{m}^{2}$ in the HA group and $33.15 \mathrm{~kg} / \mathrm{m}^{2}$ in the PRP group (Table I). The initial, I-month, and 6-month WOMAC scores in the HA group were $83.96,66.89,58.53$, respectively.

The initial, I-month, and 6-month WOMAC scores for the PRP group were 79.84, 67.81, and 64.62, respectively (Table 2).

The initial, I-month, and 6-month VAS scores in the HA group were 6.45 , 4.66, and 4.04, respectively. The initial, I-month, and 6-month VAS scores in the PRP group were 6.0, 4.21, and 3.83, respectively (Table 3 ).

There was no difference between the groups in terms of sex and Kellgren Lawrence staging. However, the duration of the complaints in the PRP group was significantly longer than that in the HA group.

In both the HA and PRP groups, pain was reduced significantly in the subsequent measurements. Thus, HA and PRP applications

\begin{tabular}{|lcccc|}
\hline \multicolumn{5}{|l|}{ TABLE I. Demographic features of the patients } \\
\hline Male & Female & Age (Mean) & BMI (Mean) \\
\hline Group HA & 6 & 22 & 63.53 & 31.48 \\
Group PRP & 6 & 26 & 63.43 & 33.15 \\
\hline \multicolumn{4}{l}{ BMI:Body Mass Index, HA: Hyaluronic acid, PRP:Platelet-rich-plasma } \\
\hline
\end{tabular}

\begin{tabular}{|c|c|c|c|}
\hline & $\begin{array}{l}\text { WOMAC score } \\
\text { (initial) }\end{array}$ & $\begin{array}{l}\text { WOMAC score } \\
\text { (at I mon) }\end{array}$ & $\begin{array}{l}\text { WOMAC score } \\
\text { (at } 6 \text { mon) }\end{array}$ \\
\hline Group HA & 83.96 & 66.89 & 58.53 \\
\hline Group PRP & 79.84 & 67.81 & 64.62 \\
\hline
\end{tabular}

\begin{tabular}{|c|c|c|c|}
\hline & $\begin{array}{l}\text { VAS score } \\
\text { (initial) }\end{array}$ & $\begin{array}{l}\text { VAS score } \\
\text { (at I mon) }\end{array}$ & $\begin{array}{l}\text { VAS score } \\
\text { (at } 6 \text { mon) }\end{array}$ \\
\hline Group HA & 6.45 & 4.66 & 4.04 \\
\hline Group PRP & 6.0 & 4.21 & 3.83 \\
\hline
\end{tabular}


were associated with a decrease in pain over time. However, no statistically significant difference was observed between the HA and PRP groups ( $p>0.05)$. When the WOMAC scores were evaluated, the function, joint stiffness, and overall WOMAC score were positively affected in both the groups of patients; however, there was no difference between the two groups ( $p>0.05$ ).

\section{DISCUSSION}

PRP and HA are both effective in knee osteoarthritis treatment and improve the patients' functionality and quality of life.

Chang et al (5) reviewed the effects of intra-articular PRP injection in knee OA compared to that of HA in a systematic review performed in 20l4. The study demonstrated that PRP caused significantly functional improvement in patients with knee cartilage pathology, where the effects lasted for at least 12 mon. Compared to patients receiving $\mathrm{HA}$, those receiving PRP had more and longer-lasting improvement. Moreover, better results were observed among patients with milder forms of OA than those whose condition was advanced.

Similar results were obtained in a 2013 meta-analysis by Khoshbin et al. (6) wherein PRP was more efficient than HA and normal saline in mild-to-moderate $O A$.

Another systematic review conducted in 2014 stated that evidence often supported the use of PRP in knee OA. Different studies have shown that PRP has an effect in causing pain relief and reducing the clinical symptoms in 6 months. However, there is no evidence advocating PRP efficiency in traumatic or degenerative chondral pathology. Therefore, high-quality randomized controlled trial studies are warranted to compare PRP with placebo and surgical treatments supplemented by PRP with operative management alone (7).

In a meta-analysis conducted by Merchan in 2013 (8), the efficiencies of steroids, HA, and PRP were reviewed. The researchers suggested 3-5 weekly HA injections in the OA knee before performing surgical treatment. They concluded that steroid injections had very short-term effects; however, PRP injections needed to be further investigated to determine the grade and duration of the efficiency.

In another study by Vaquerizo et al. (9) in 2013, 96 patients in two groups received three therapy injections of plasma rich in growth factors (PRGF) or a single injection of HA and were followed up for $48 \mathrm{wk}$. The efficiency of PRGF in terms of pain and stiffness decrement and physical performance improvement was greater than that of HA. In addition, the patients' responses to PRGF in all scores, including those for pain, stiffness, and physical performance in the WOMAC, Lequesne, and Outcome Measures in Rheumatology Osteoarthritis Research Society International were more meaningful than those for $\mathrm{HA}$; this result is contradictory to our finding.

Filardo et al (IO) conducted a study in 2012 to compare PRP and $\mathrm{HA}$ in the treatment of knee OA. Total 109 patients (55 in the HA group and 54 in the PRP group) participated in the study. They were evaluated at the beginning and at 2, 6, and 12 months after the treatment using the Knee injury and Osteoarthritis Outcome Score, International Knee Documentation Committee (IKDC), and EuroQol (EQ)-VAS questionnaires. PRP/HA were injected thrice with one-week intervals. At the end of the follow-up, significant improvements were observed in all the parameters in both the groups. However, there were no meaningful differences between the groups in terms of the EQ-VAS and IKDC scores. The authors concluded that PRP should not be given priority over HA in middle-aged patients with moderate OA and should not be applied as the first-line treatment.

Filardo et al. (II) evaluated the benefits provided by PRP and $\mathrm{HA}$ injections in the treatment of knee-joint degeneration. They concluded that PRP does not provide a superior clinical improvement than HA. In a similar manner, in our study, when the WOMAC scores were evaluated, the function, joint stiffness, and overall WOMAC scores were positively affected in both the groups; however, there were no between-group differences.

A meta-analysis by Sadabad et al. (12) investigated the efficiency of PRP and HA in the treatment of knee osteoarthritis. Seven studies on 722 subjects ( 364 in the PRP group and 358 in the HA group) were analyzed. The results of this meta-analysis two years after the PRP injection showed the efficacy of PRP versus that of HA.

Cerza et al (13) compared the clinical response of HA and PRP treatment in the two groups of patients affected by gonarthrosis. They conclude that treatment with HA did not seem to be effective in patients with grade III gonarthrosis.

The limitations of this study include the absence of a placebo control group, lack of blinding, and the lack of objective evaluation of the treatment effects on the morphology of the cartilage, soft tissue, and other intra- and peri-articular structures of the knee. Furthermore, considering the higher cost of PRP than that of other injection therapies, such as HA, and the need for special kit and a centrifuge devise for using PRP, the administration of this therapy should be carefully considered (cost-effectiveness and availability).

In addition, this study was performed retrospectively. Long-term prospective studies may be helpful to consider more detailed knowledge about this topic.

\section{CONCLUSION}

PRP is a novel option in knee OA management, and an increasing number of clinical studies have shown promising results. Both PRP and HA have positive effects in early gonarthrosis; however the results indicated no superiority in the PRP group.

Ethics Committee Approval: Ethics committee approval was received for this study from the ethics committee of of Dokuz Eylul University, 2017.

Informed Consent: Due to the retrospective design of the study, informed consent was not taken.

Peer-review: Externally peer-reviewed.

Author contributions: Concept - Design-Y.E.B., O.G, N.D.D.; Supervision Y.E.B, N.D.D.; Resource - Y.E.B, O.G.; Materials - Y.E.B, O.G, N.D.D.; Data Collection and/or Processing - Y.E.B, O.G, N.D.D.; Analysis and/or Interpretation - Y.E.B, O.G., N.D.D.; Literature Search-Y.E.B, O.G, N.D.D, R.Ö.; Writing - Y.E.B, N.D.D, R.Ö; Critical Reviews - N.D.D, R.Ö. 
Conflict of Interest: The authors have no conflicts of interest to declare.

Financial Disclosure: The authors declared that this study has received no financial support.

\section{REFERENCES}

I. Wong LY, Yiu RL, Chiu CK, Lee WK, Lee YL, Kwong PK, et al. Prevalence of Psychiatric Morbidity in Chinese Subjects with Knee Osteoarthritis in a Hong Kong Orthopaedic Clinic East Asian Arch Psychiatry 2015; 25(4): 150-8.

2. Neogi T. The epidemiology and impact of pain in osteoarthritis. Osteoarthritis Cartilage 2013; 2I(9): ||45-53. [CrossRef]

3. Michael JW, Schlüter-Brust KU and Eysel P. The epidemiology, etiology, diagnosis and treatment of osteoarthritis of the knee. Dtsch Arztebl Int 2010; 107(9): 152-62. [CrossRef]

4. Sinusas K. Osteoarthritis: diagnosis and treatment. Am Fam Physician 2012; 85(I): 49-56.

5. Chang KV, Hung CY, Aliwarga F, Wang TG, Han DS, Chen WS, et al. Comparative effectiveness of platelet-rich plasma injections for treating knee joint cartilage degenerative pathology: a systematic review and meta-analysis. Arch Phys Med Rehabil_2014; 95(3): 56275. [CrossRef]

6. Khoshbin A, Leroux T, Wasserstein D, Marks P, Theodoropoulos J, Ogilvie-Harris $D$, et al. The efficacy of platelet-rich plasma in the treatment of symptomatic knee osteoarthritis: a systematic review with quantitative synthesis. Arthroscopy 2013; 29(12): 2037-48. [CrossRef]
7. Dold AP, Zywiel MG, Taylor DW, Dwyer T, Theodoropoulos J. Platelet-rich plasma in the management of articular cartilage pathology: a systematic review. Clin J Sport Med 20I4; 24(I): 31-43. [CrossRef]

8. Rodriguez-Merchan EC. Intra-articular Injections of Hyaluronic Acid and Other Drugs in the Knee Joint HSS J 20I3; 9(2): 180-2. [CrossRef]

9. Vaquerizo V, Plasencia MÁ, Arribas I, Seijas R, Padilla S, Orive G, et al. Comparison of intra-articular injections of plasma rich in growth factors (PRGF-Endoret) versus Durolane hyaluronic acid in the treatment of patients with symptomatic osteoarthritis: a randomized controlled trial. Arthroscopy 2013; 29(I0): 1635-43. [CrossRef]

10. Filardo G, Kon E, Di Martino A, Di Matteo B, Merli ML, Cenacchi A, et al. Platelet-rich plasma vs hyaluronic acid to treat knee degenerative pathology: study design and preliminary results of a randomized controlled trial. BMC Musculoskelet Disord 2012; 23; 13:229. [CrossRef]

II. Filardo G, Di Matteo B, Di Martino A, Merli ML, Cenacchi A, Fornasari $\mathrm{P}$, et al. Platelet-Rich Plasma Intra-articular Knee Injections Show No Superiority Versus Viscosupplementation: A Randomized Controlled Trial. Am J Sports Med 2015; 43(7): 1575-82. [CrossRef]

12. Sadabad HN, Behzadifar M, Arasteh F, Behzadifar M, Dehghan HR Efficacy of Platelet-Rich Plasma versus Hyaluronic Acid for treatment of Knee Osteoarthritis: A systematic review and meta-analysis. Electronic Physician 2016; 8(3): 2115-22. [CrossRef]

13. Cerza F, Carnì S, Carcangiu A, Di Vavo I, Schiavilla V, Pecora A, et al. Comparison between hyaluronic acid and platelet-rich plasma, intra-articular infiltration in the treatment of gonarthrosis. Am J Sports Med 2012; 40(12): 2822-7. [CrossRef] 